\title{
Tunable 2D binary colloidal alloys for soft nanotemplating†
}

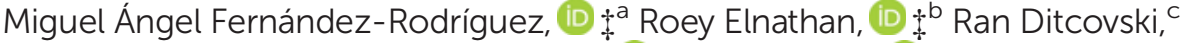 \\ Fabio Grillo, ${ }^{a}$ Gaurasundar Marc Conley, (D) d Flavia Timpu, (D) e Astrid Rauh, \\ Karen Geisel, ${ }^{g}$ Tal Ellenbogen, ${ }^{c}$ Rachel Grange, (D) e Frank Scheffold, (D) d \\ Matthias Karg, (iD ${ }^{f}$ Walter Richtering, (i) ${ }^{g}$ Nicolas H. Voelcker (iD ${ }^{b, h, i}$ and Lucio Isa (iD) *a
}

The realization of non-close-packed nanoscale patterns with multiple feature sizes and length scales via colloidal self-assembly is a highly challenging task. We demonstrate here the creation of a variety of tunable particle arrays by harnessing the sequential selfassembly and deposition of two differently sized microgel particles at the fluid-fluid interface. The two-step process is essential to achieve a library of 2D binary colloidal alloys, which are kinetically inaccessible by direct co-assembly. These versatile binary patterns can be exploited for a range of end-uses. Here we show that they can for instance be transferred to silicon substrates, where they act as masks for the metal-assisted chemical etching of binary arrays of vertically aligned silicon nanowires (VA-SiNWs) with fine geometrical control. In particular, continuous binary gradients in both NW spacing and height can be achieved. Notably, these binary VA-SiNW platforms exhibit interesting anti-reflective properties in the visible range, in agreement with simulations. The proposed strategy can also be used for the precise placement of metallic nanoparticles in non-close-packed arrays. Sequential depositions of soft particles enable therefore the exploration of complex

${ }^{a}$ Laboratory for Interfaces, Soft matter and Assembly, Department of Materials, ETH Zurich, 8093 Zurich, Switzerland. E-mail: lucio.isa@mat.ethz.ch

${ }^{b}$ Drug Delivery, Disposition and Dynamics, Monash Institute of Pharmaceutical Sciences Monash University, 381 Royal Parade, Parkville, Victoria, 3052, Australia ${ }^{c}$ Department of Physical Electronics, School of Electrical Engineering,

Tel Aviv University, Tel Aviv-Yafo, Israel

${ }^{d}$ Department of Physics, University of Fribourg, $\mathrm{CH}-1700$ Fribourg, Switzerland ${ }^{e}$ Institute for Quantum Electronics, Department of Physics, ETH Zurich, 8093 Zurich, Switzerland

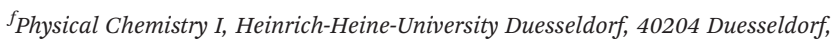
Germany

${ }^{g}$ Physical Chemistry II, RWTH Aachen University, 52062 Aachen, Germany ${ }^{h}$ Melbourne Centre for Nanofabrication, Victorian Node of the Australian National Fabrication Facility, 151 Wellington Road, Clayton, Victoria, 3168, Australia ${ }^{i}$ INM-Leibniz Institute for New Materials, Campus D2 2, Saarbrücken, 66123, Germany

$\dagger$ Electronic supplementary information (ESI) available. See DOI: 10.1039/ c8nr07059h

$¥$ These authors contributed equally to this work. binary patterns, e.g. for the future development of substrates for biointerfaces, catalysis and controlled wetting.

Crystalline monolayers from binary mixtures of colloidal particles (2D colloidal alloys) freely floating at a fluid-fluid interface constitute ideal model systems to study the structure and dynamics of materials, ${ }^{1-6}$ but they become even more interesting for applications after they are transferred onto solid supports. ${ }^{7}$ For instance, they can be used as photonic crystals with combined band gaps, ${ }^{8}$ to produce superhydrophobic surfaces thanks to their hierarchical features, ${ }^{9}$ to realize plasmonic materials when noble-metal particles are used, ${ }^{10}$ or for enhanced electronic properties when semiconducting ones are employed. ${ }^{11}$ Additionally, versatile binary patterns can also be highly beneficial for the realization of vertically aligned silicon nanowire arrays (VA-SiNWs) for catalysis, ${ }^{12}$ energy conversion and storage,$^{13}$ and to engineer nano-bio cellular interfaces. ${ }^{14-16}$

For both fundamental studies and applications, full control on the particle size, separation and arrangement is highly desirable. Nevertheless, practically all fabrication strategies for 2D binary colloidal alloys present a major constraint: all the particles are in direct contact after deposition on the solid substrate. As a consequence, the interparticle distance cannot be independently and continuously changed relative to the particle size, especially over the same substrate. This is the case for both one-step methods, e.g. where binary particle populations are co-injected and deposited from a fluid interface, ${ }^{17,18}$ or two-step methods, where particles of different sizes or material are deposited onto a substrate one after the other, e.g. by spin-coating, dip-coating or capillary assembly. ${ }^{8,9,19,20}$ In these cases, typical structures display compact arrays of the larger colloids with the small particles in the interstices. A range of strategies has been proposed to achieve non-close-packed single-component crystalline monolayers, ${ }^{21-26}$ but the possibilities to produce tunable, nonclose-packed binary arrays remains practically untapped.

We report here a two-step method for the fabrication of tunable 2D binary colloidal alloys based on sequential 
depositions of Langmuir monolayers of soft core-shell poly $(N-$ iso-propylacrylamide) (PNIPAM) microgels of different sizes onto a silicon substrate. By simultaneously compressing a particle-laden water/hexane interface and lifting a silicon wafer through it, we fabricate complex microgel binary arrays, achieving independent control on the size and separation of each particle type. We furthermore demonstrate the process using a variety of different soft microgel-based nanoparticles (Fig. 1a, and Fig. S1 in the ESI†). In particular, we use two differently sized PNIPAM microgels with bulk hydrodynamic diameters of
$940 \mathrm{~nm}$ and $426 \mathrm{~nm}$, hereon termed 'big' and 'small' microgels, and core-shell nanoparticles with a gold core of $75 \mathrm{~nm}$ embedded in a PNIPAM shell with a bulk hydrodynamic diameter of $340 \mathrm{~nm}$, hereon termed 'gold core-shell' particles (Fig. 1b).

One-step routes, i.e. pre-mixing the big and small microgels before confining them at the water/hexane interface or simultaneously co-injecting them at the interface from two separate syringes, do not lead to the realization of uniform binary lattices (Fig. S2 and S3 in the ESI $\dagger$ ). In both cases, the big
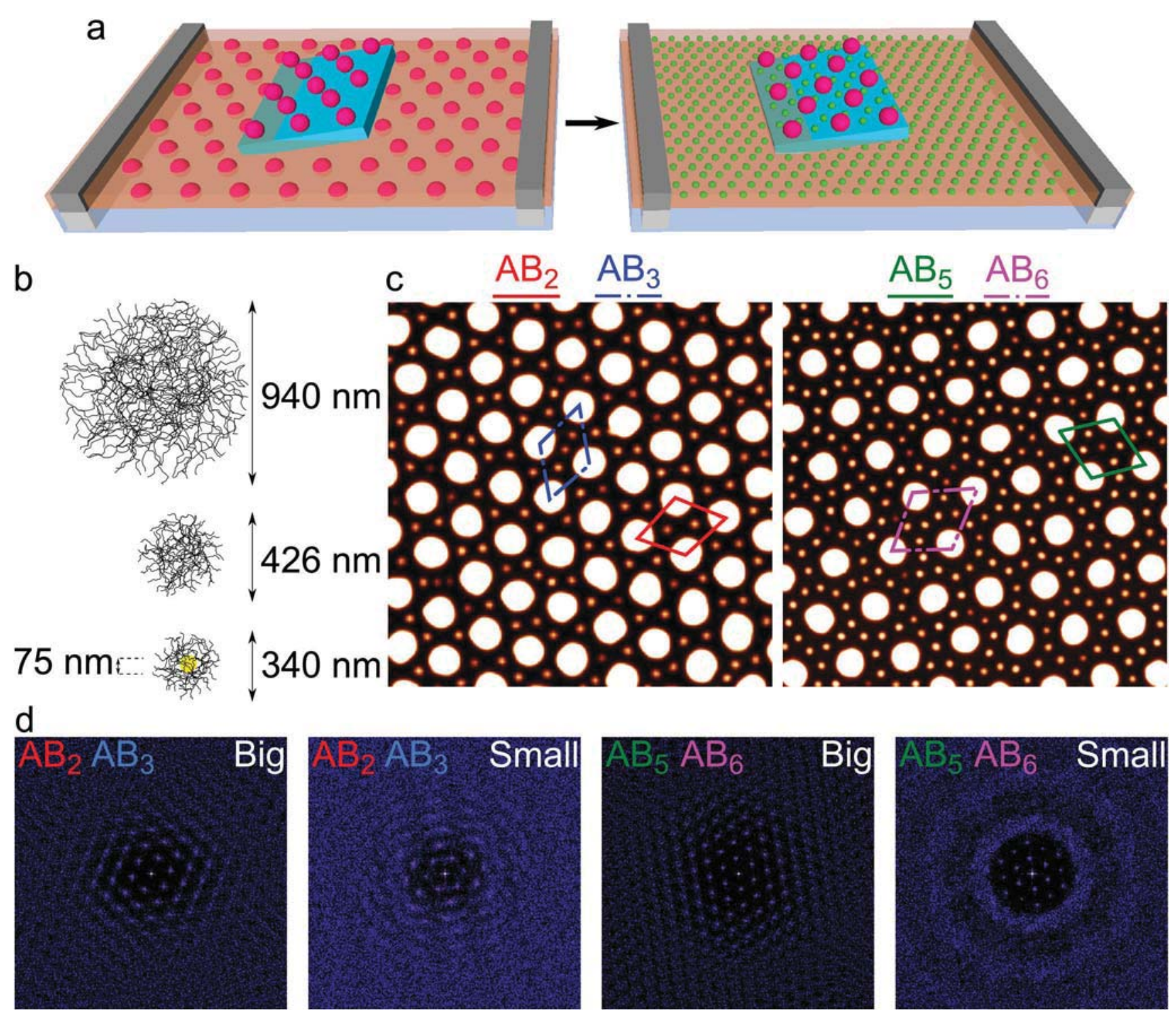

Fig. 1 (a) Scheme of the sequential deposition technique at the water/hexane interface: first (left scheme), the big microgels are deposited from the interface by lifting the silicon substrate and controlling the interfacial pressure $\Pi_{\text {big }}$ using the barriers of the Langmuir trough. In a second step (right scheme), the same substrate is immersed in water and a second layer of small microgels is deposited from the interface, with controlled $\Pi_{\text {small }}$. (b) Scheme of the three types of soft core-shell PNIPAM microgels used in this study: big, small and gold core-shell particles. (c) AFM images (10 $x$ $10 \mu \mathrm{m}^{2}$ ) showing different microstructures of deposited binary arrays with varying interparticle distances between the big and small microgels (left: $\Pi_{\text {big }}=4 \mathrm{mN} \mathrm{m}^{-1}$ and $\Pi_{\text {small }}=5 \mathrm{mN} \mathrm{m}^{-1}$, right: $\Pi_{\text {big }}=2 \mathrm{mN} \mathrm{m}^{-1}, 20 \mathrm{~s}$ of $\mathrm{O}_{2}$ plasma etching and $\Pi_{\text {small }}=2 \mathrm{mN} \mathrm{m}^{-1}$ ). Four types of unit cells of $2 \mathrm{D}$ binary colloidal alloys are highlighted, where the stoichiometry of big and small microgels is represented by $A_{B_{x}}$, respectively. The height range of the AFM images goes from 0 to $50 \mathrm{~nm}$ to allow for the simultaneous visualization of both particles. (d) FFT of the positions of the microgels in (c), separating big and small microgels. The FFTs were calculated for the larger $20 \times 20 \mu \mathrm{m}^{2}$ images corresponding to the ones shown in (c). 
microgels form domains or aggregates, driven by capillary attraction, ${ }^{27}$ from which the small microgels are excluded. Therefore, we developed a two-step route where the two types of microgels are injected at the interface, compressed and transferred onto the substrate via two independent depositions in succession. As a starting point, we first characterized the compression of single-component interfacial monolayers of the big and small microgels alone in a Langmuir trough. Upon adsorption at the water/hexane interface, microgels deform and flatten out, ${ }^{28}$ acquiring a cross-sectional diameter at the interface 1.7 and 2.6 times larger than their bulk diameter, for the big and small microgels, respectively (Table $\mathrm{S} 2 \dagger$ for details). Furthermore, as previously reported for similar particles, ${ }^{27-30}$ they take up a pronounced $2 \mathrm{D}$ core-shell morphology. In fact, during synthesis, these microgels develop a varying crosslinking density profile, with a denser core surrounded by a fuzzy shell. Once at the interface, the shell forms a deformable corona surrounding the core within the interface plane, so that steric interactions are first mediated through the shells before the cores can come into contact. Upon compression, both particle types show a large range of surface pressures for which 2D long-range ordered hexagonal crystalline monolayers with tunable lattice constants are found (from 1 to $22 \mathrm{mN} \mathrm{m}^{-1}$ for small and from 1 to $10 \mathrm{mN} \mathrm{m}^{-1}$ for big microgels, Fig. $\mathrm{S} 1 \dagger) .{ }^{28,29}$ These regions correspond to particles in compressible shell-shell contacts, where the distance between microgel cores can be tuned independently from the overall (core + shell) microgel size. These regions define the working parameter space for the realization of the binary arrays. In fact, we then proceeded to their realization by first depositing hexagonal arrays of the big microgels, followed by the deposition of arrays of the small microgels, both at controlled surface pressures, on the same substrate (Fig. 1a). Full details of the deposition protocols are found in the ESI. $\dagger$

At this stage, several observations can be made. During the second deposition step, the arrays of big microgels deposited during the first step were re-immersed into water and passed through the water/hexane interface again. We first checked that the big microgels were rehydrated upon water immersion, therefore providing soft steric interactions with the coming monolayer of small particles. Then, most importantly, we demonstrated that adhesion with the substrate prevents any significant displacement of the first array of big microgels due to the capillary forces experienced by the particles when crossing the interface for the second time (Fig. S4 and Movie S1 in the ESI $\dagger$ ). This implies that the interparticle distance between the big microgels could be considered fixed during the second step. We could therefore independently control the distance between big and small microgels through the respective compressions at the water/hexane interface, producing different relative densities of the two particles.

Examples of these sequential depositions, displaying a range of $2 \mathrm{D}$ binary colloidal alloy lattices $\mathrm{AB}_{x}$, are shown in Fig. 1c. Crucially, and differently to two-steps methods described above, the small microgels form regular arrays in the interstices between the big ones, but they are on the same plane. Moreover, due to the softness of the interactions, the separation between small and big microgels can be freely tuned, by "squeezing" more of them in the interstitial spaces. The area per particle of the small microgels obtained for a given surface pressure during the second deposition is different from the one obtained when no big microgels are deposited in the first place (Fig. S5a and $b \dagger$ ). The steric interactions with the big microgels in fact limit the available space for the smaller ones to the interstices and practically no deposition of the second microgels on top of the first ones is seen (Fig. S5c $\dagger$ ). The different structures observed in Fig. 1c are defined by the number ratio of the two different microgels. In order to describe the structural feature of the patterns further, Fig. 1d shows the Fourier transforms (FFTs) of the patterns corresponding to the $\mathrm{AB}_{2}-\mathrm{AB}_{3}$ region and the $\mathrm{AB}_{5}-\mathrm{AB}_{6}$ region, reporting the FFTs of the two particle populations separately. In both cases, the presence of a long-range hexagonal order for the array of the big microgels is evident. Since these two samples were prepared with the same compression of the big microgels, the peak positions are the same and the two Fourier patterns are rotated reflecting a different grain orientation. Interestingly, when examining the FFTs of the small particles alone, we see that the global hexagonal order imposed by the large microgels is maintained, while the local orientational order is quickly lost. Compression of the second array has therefore the main effect of controlling the interparticle distance.

A key advantage of the sequential deposition is thus the generation of continuous and decoupled gradients of particle density on the same substrate. For instance, we can compress the small microgels to deposit up to five of them between each two big ones, while the interparticle distance between the big microgels is fixed, as it can be seen in Fig. 2a. This is quantitatively shown in Fig. 2b. After extracting the positions of all particles from AFM images, we can measure their separation. In particular, for the sample shown in Fig. 2a, the separation between big microgels was fixed to $1.5 \mu \mathrm{m}\left(2 \mathrm{mN} \mathrm{m}^{-1}\right.$ constant surface pressure), while the separation between small microgels varied from $450 \mathrm{~nm}$ to $250 \mathrm{~nm}$ (surface pressure varying between 3 to $22 \mathrm{mN} \mathrm{m}^{-1}$ ), with excellent control over the entire $2 \times 2 \mathrm{~cm}^{2}$ substrate. Additional information on the local structure can be obtained by measuring local bond order parameters. Fig. 2c shows the hexagonal bond order parameter $\Psi_{6}$ for the big particles as a function of position along the gradient sample shown in Fig. 2a. The values are consistently close to 1 , indicating a high degree of hexagonal order over the whole substrate. For the small particles, we instead show both $\Psi_{6}$ and $\Psi_{3}$, the three-fold coordination order parameter (the definitions of the order parameters are found in the ESI $\dagger$ ). As it can be qualitatively seen in the AFM images, at the beginning of the gradient the small particles surround the big ones in a single row, so that a triangular coordination emerges with $\Psi_{3}>\Psi_{6}$. Upon compression, the small particles are packed into multiple files between the big ones and they locally acquire a hexagonal packing, as shown by the cross-over to a region where $\Psi_{6}>\Psi_{3}$. Further characterization of these arrays 


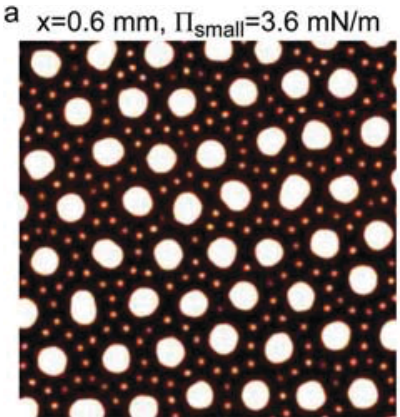

b

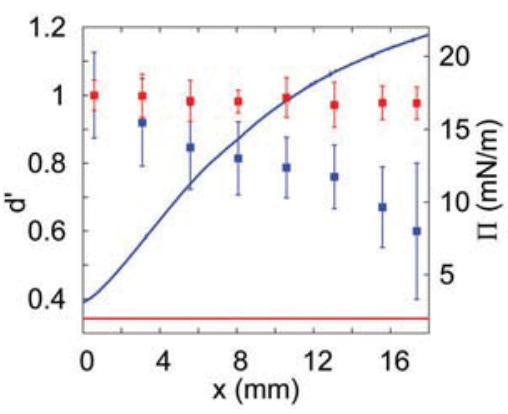

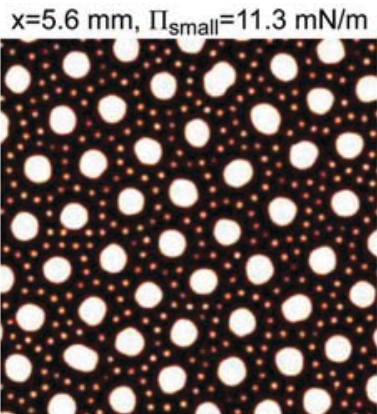

C

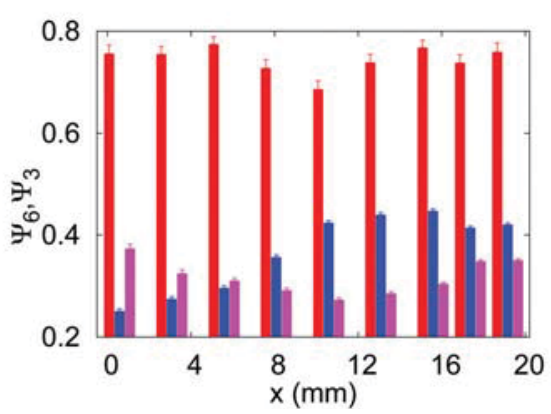

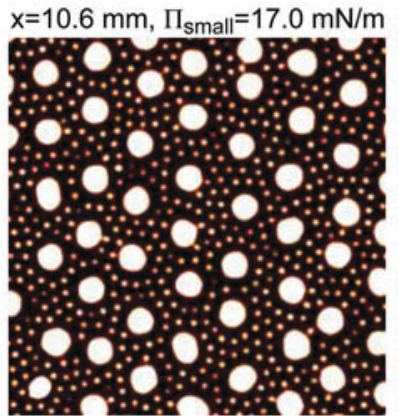

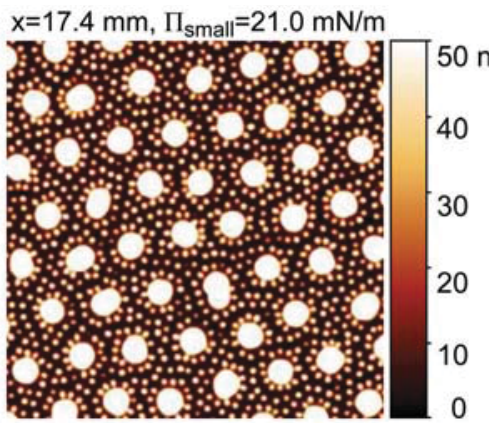

d

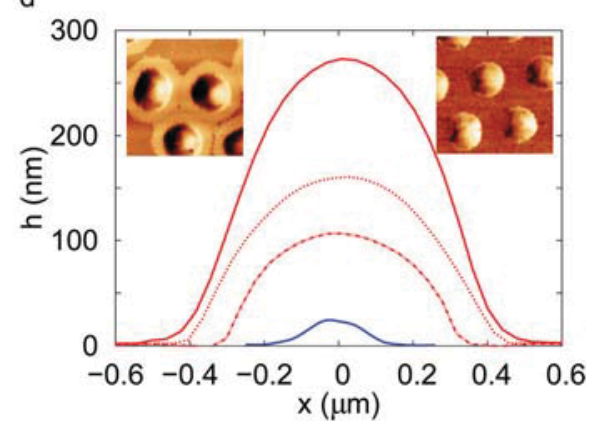

Fig. 2 (a) AFM height images $\left(10 \times 10 \mu \mathrm{m}^{2}\right.$, height scale is limited to $50 \mathrm{~nm}$ to show both microgels $)$ of sequential depositions with $\Pi_{\text {big }}=2 \mathrm{mN} \mathrm{m}^{-1}$ and compression of the small microgels from $\Pi_{\text {small }}=3$ to $22 \mathrm{~m} \mathrm{~N} \mathrm{~m}^{-1}$ at different positions $x$ over the wafer, with increasing interfacial pressure

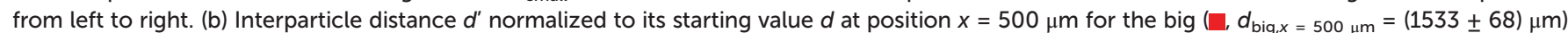
and small $\left(\square, d_{\text {small }, x}=500 \mu m=(437 \pm 55) \mu \mathrm{m}\right)$ microgels for the same substrate in (a). The surface pressure versus distance is also plotted for both sequential depositions ( - and - for the first and second deposition, respectively). (c) Bond orientation order parameters $\Psi_{6}$ for the big (red) and small microgels (blue) and $\Psi_{3}$ for the small microgels (magenta), for the same substrate in (a). (d) Height profiles from AFM images of the big (-) and small (-) microgels. The effect of plasma on the big microgels can be seen as the size of the big microgel decreases with increasing plasma treatment times: $120 \mathrm{~s}(\cdot .$.$) and 500 \mathrm{~s}(-\cdot-)$. The insets are AFM phase images $\left(3 \times 3 \mu \mathrm{m}^{2}\right)$ before (left) and after (right) $30 \mathrm{~s}$ of oxygen plasma treatment. By comparing the left and right images, we show that the shell has disappeared after the short plasma treatment.

is given in Fig. S6 in the ESI, $\dagger$ where together with the FFTs, the autocorrelation of the orientational bond order parameters is given, emphasizing that structural order is long-ranged for the big microgels and short-ranged for the small ones.

Different binary samples can therefore be made using different combinations of compressions (e.g. compressing the big microgels and keeping the small ones at a fixed surface pressure, as shown in Fig. S7 in the ESI $\dagger$ ) demonstrating the greater flexibility and robustness of our method. A further degree of freedom in tuning the geometrical parameters was achieved by controlled oxygen-plasma etching of the microgels, which is used to vary their size (Fig. 2d and Fig. S8 in the ESI $\dagger$ ). Very short plasma times (i.e. $30 \mathrm{~s}$ ) had the effect of removing only the particle shells, enabling the possibility to tailor the interactions between microgels during the first and the second step of deposition, while more prolonged plasma times led to a controlled reduction of the particle diameter.

As a first example of a potential use for the binary microgel arrays described here, we show their application as nanolithography masks. After depositing the desired binary colloidal alloy on a silicon substrate, we swelled the microgels with photoresist to increase their thickness (Fig. S9 in the ESI $\dagger$ ) and sputter-coated them with a $10 \mathrm{~nm}$-thick gold layer. This process converted the microgels into effective masks for the metal-assisted chemical etching (MACE) of the silicon wafers in a customized etching solution to produce binary arrays of VA-SiNWs (Fig. 3a, b and Fig. S10 in the ESI†. Details on the production and characterization of VA-SiNWs are in the ESI $\dagger$ ). ${ }^{14,30,31}$ During the process, we further demonstrated fine control over the NW height through dipping the substrate in the etching solution using a linear motion drive and smoothly varying the etching time along the sample (Fig. 3c, Fig. S11 and details on the height gradients in the ESI $\dagger$ ). Building on these results, we achieved independent control over three key parameters in the fabrication of binary SiNW arrays: (i) NW separation can be controlled during the sequential deposition, (ii) NW diameter is controlled by oxygen plasma etching, potentially after each deposition step, and (iii) NW height is controlled by the etching time.

A platform based on binary VA-SiNW arrays is a promising avenue to realize materials for a range of applications, including controlled wetting, engineered cellular interactions and targeted light-nanostructure interactions. As an example, we in particular show here that the binary arrays have interesting anti-reflective properties in the visible range. We report the optical normal reflectance of binary arrays of VA-SiNWs both from finite-difference-time-domain simulations and experiments, and compared them to arrays of VA-SiNWs made using 

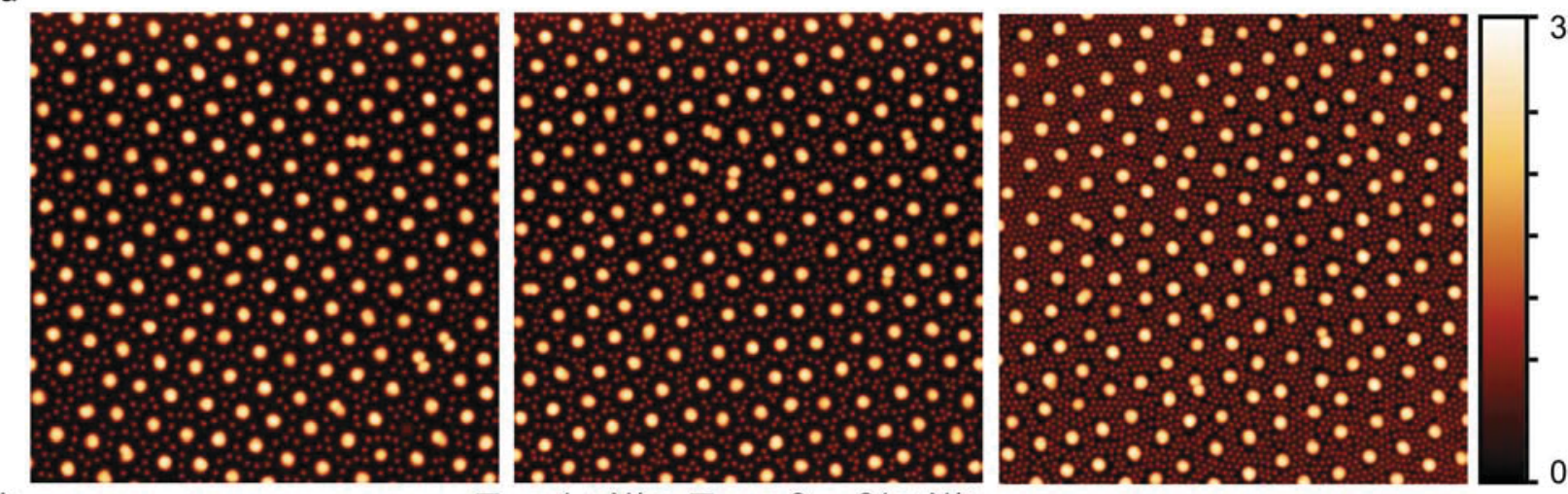

$380 \mathrm{~nm}$

b

$\Pi_{\text {big }}=1 \mathrm{mN} / \mathrm{m}, \Pi_{\text {small }}=2 \rightarrow 21 \mathrm{mN} / \mathrm{m}$
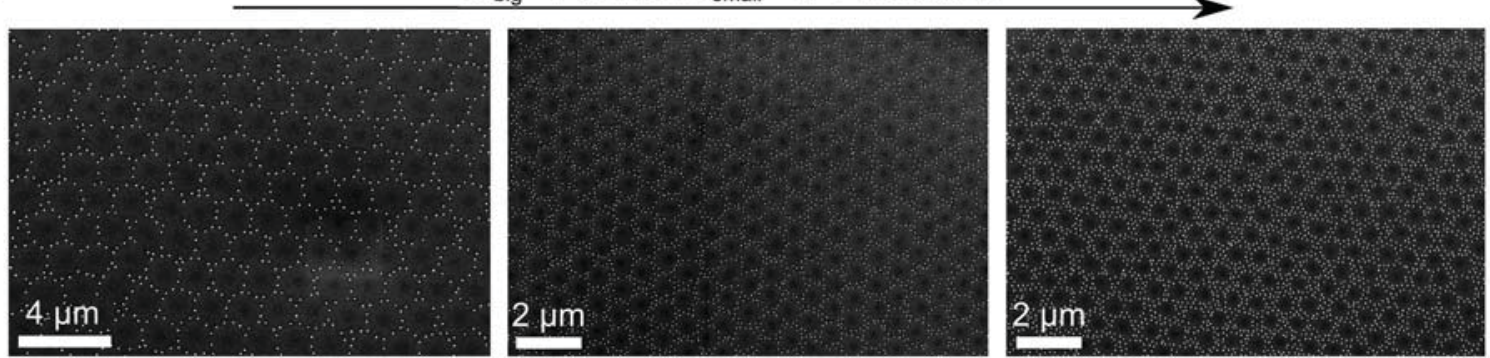

$2 \mu \mathrm{m}$

Fig. 5 (a) $20 \times 20 \mu \mathrm{m}^{2}$ AFM height images from a decoupled continuous gradient, where the big microgels were kept at a constant surface pressure value of $1 \mathrm{mN} \mathrm{m}^{-1}$, while gold-core small microgels were compressed from 2 to $21 \mathrm{mN} \mathrm{m}^{-1}$ from left to right. (b) Honeycomb gold nanoparticle patterns on the silicon substrate obtained after $30 \mathrm{~min}$ of $\mathrm{O}_{2}$ plasma etching of the substrate in (a).

only big microgels (Fig. 4). The experimental spectra are the average of three measurements taken over different parts of the same substrate. Details of the simulations and experimental procedures are in the ESI, and Fig. S12, $\dagger$ together with the full simulated spectra for a broad range of NW heights from $400 \mathrm{~nm}$ to $12 \mu \mathrm{m}$. The simulated spectra for the arrays with big particles only show that there are transverse resonances of the NWs in the visible range (i.e. $\lambda$ from $390 \mathrm{~nm}$ to $750 \mathrm{~nm})$, that weakly depend on the NW height. The experimental spectra reproduce the qualitative features of the simulated spectra, but with a broadening of the spectral peaks, due to polydispersity in the NW geometrical parameters and interspacing compared to the simulation (details of this effect are given in Fig. S13 in the ESI $\dagger$ ). The most striking feature of the spectra for the binary NW arrays is that the overall reflectance is greatly reduced by the presence of the smaller NWs, indicating the possible use of the binary arrays for anti-reflective coatings. This effect is particularly pronounced for the longer NWs, while the shorter NWs are specifically reducing reflectance around 400 and $700 \mathrm{~nm}$. The dark-field reflectance is also reduced by the presence of the smaller NWs, that partly disrupt the grating effect caused by the bigger NWs (see Fig. S13†). By varying the dimensions of the wires one can expect to tune the anti-reflective properties of the patterned surfaces.

To demonstrate further the potential of our sequential fabrication method as a versatile nanopatterning tool, we used the gold core-shell nanoparticles instead of the small micro- gels to form the binary lattices (Fig. 5a). Upon removing all the organic material via $30 \mathrm{~min}$ of $\mathrm{O}_{2}$ plasma (Fig. 5b), the binary microgel arrays are transformed into honeycomb lattices of the gold cores. By tuning the compression of the gold core-shell microgels, we produced patterns with increasing density of metallic nanoparticles. These patterns might be interesting for a variety of applications, including the realization of plasmonic surfaces with fine and continuous tuning of the lattices.

To conclude, this work shows that it is possible to preprogram sequential assemblies of various types of microgels at fluid interfaces, transfer them onto silicon substrates and thus realize complex non-close-packed binary nanopatterns. We showed that the transferred structures can be used as lithographical etching masks, enabling the controlled growth of binary nanowire arrays, with combinatorial control on their geometrical parameters, including their height, using MACE. The same assembly and patterning strategy can be applied to the realization of additional nanostructures. We expect that the extension of sequential particle assembly and deposition routes harnessing fluid interfaces will open up many interdisciplinary opportunities for the realization of complex nanopatterns for applications in optics, material science, sensing and nano-bio cellular interfaces.

\section{Conflicts of interest}

There are no conflicts to declare. 


\section{Acknowledgements}

Authors thank N. D. Spencer, A. Studart and M. Fiebig for access to instrumentation. L. I., R. E. and M. A. F. R acknowledge the financial support of the Swiss National Science Foundation Grants PP00P2_144646/1, PP00P2 172913/1 and IZK0Z2_171156 and of the Swiss Government Excellence Postdoc Scholarship 2016.0246. K. G. and W. R. thank the German Research Foundation (Collaborative Research Center SFB985). R. E acknowledge the Australian Research Council for his DECRA (DE170100021). This work was performed in part at the Melbourne Centre for Nanofabrication (MCN) in the Victorian Node of the Australian National Fabrication Facility (ANFF).

\section{References}

1 A. D. Law, D. M. A. Buzza and T. S. Horozov, Phys. Rev. Lett., 2011, 106, 128302.

2 J. Fornleitner, F. Lo Verso, G. Kahl and C. N. Likos, Soft Matter, 2008, 4, 480-484.

3 L. Assoud, F. Ebert, P. Keim, R. Messina, G. Maret and H. Löwen, J. Phys.: Condens. Matter, 2009, 21, 464114.

4 F. Ebert, G. Maret and P. Keim, Eur. Phys. J. E: Soft Matter Biol. Phys., 2009, 29, 311-318.

5 S. Mazoyer, F. Ebert, G. Maret and P. Keim, Eur. Phys. J. E, 2011, 34, 101.

6 L. J. Bonales, F. Martínez-Pedrero, M. A. Rubio, R. G. Rubio and F. Ortega, Langmuir, 2012, 28, 16555-16566.

7 N. Vogel, M. Retsch, C.-A. Fustin, A. del Campo and U. Jonas, Chem. Rev., 2015, 115, 6265-6311.

8 D. Wang and H. Möhwald, Adv. Mater., 2004, 16, 244247.

9 G. Zhang, D. Wang, Z.-Z. Gu and H. Möhwald, Langmuir, 2005, 21, 9143-9148.

10 T. Honold, K. Volk, M. Retsch and M. Karg, Colloids Surf., A, 2016, 510, 198-204.

11 J. J. Urban, D. V. Talapin, E. V. Shevchenko, C. R. Kagan and C. B. Murray, Nat. Mater., 2007, 6, 115.

12 M. Xie, X. Xiong, L. Yang, X. Shi, A. M. Asiri and X. Sun, Chem. Commun., 2018, 54, 2300-2303.
13 N. Fukata, T. Subramani, W. Jevasuwan, M. Dutta and Y. Bando, Small, 2017, 13, 1701713.

14 R. Elnathan, B. Delalat, D. Brodoceanu, H. Alhmoud, F. J. Harding, K. Buehler, A. Nelson, L. Isa, T. Kraus and N. H. Voelcker, Adv. Funct. Mater., 2015, 25, 7215-7225.

15 R. Elnathan, L. Isa, D. Brodoceanu, B. Delalat, F. Harding, T. Kraus and N. Voelcker, ACS Appl. Mater. Interfaces, 2015, 7, 23717-23724.

16 J.-H. Lee, A. Zhang, S. You and C. Lieber, Nano Lett., 2016, 16, 1509-1513.

17 Z. Dai, Y. Li, G. Duan, L. Jia and W. Cai, ACS Nano, 2012, 6, 6706-6716.

18 J. Yu, Q. Yan and D. Shen, ACS Appl. Mater. Interfaces, 2010, 2, 1922-1926.

19 M. Kim, S. Im and O. Park, Adv. Mater., 2005, 17, 25012505.

20 V. Lotito and T. Zambelli, Langmuir, 2016, 32, 9582-9590.

21 K. Volk, J. P. Fitzgerald, M. Retsch and M. Karg, Adv. Mater., 2015, 27, 7332-7337.

22 L. Isa, K. Kumar, M. Müller, J. Grolig, M. Textor and E. Reimhult, ACS Nano, 2010, 4, 5665-5670.

23 C. Haginoya, M. Ishibashi and K. Koike, Appl. Phys. Lett., 1997, 71, 2934-2936.

24 M. Ray and L. Jia, Adv. Mater., 2007, 19, 2020-2022.

25 X. Li, T. Wang, J. Zhang, X. Yan, X. Zhang, D. Zhu, W. Li, X. Zhang and B. Yang, Langmuir, 2010, 26, 2930-2936.

26 J.-T. Zhang, L. Wang, J. Luo, A. Tikhonov, N. Kornienko and S. A. Asher, J. Am. Chem. Soc., 2011, 133, 9152-9155.

27 L. Scheidegger, M. A. Fernandez-Rodriguez, K. Geisel, M. Zanini, R. Elnathan, W. Richtering and L. Isa, Phys. Chem. Chem. Phys., 2017, 19, 8671-8680.

28 K. Geisel, L. Isa and W. Richtering, Langmuir, 2012, 28, 15770-15776.

29 M. Rey, M. A. Fernandez-Rodriguez, M. Steinacher, L. Scheidegger, K. Geisel, W. Richtering, T. M. Squires and L. Isa, Soft Matter, 2016, 12, 3545-3557.

30 M. Rey, R. Elnathan, R. Ditcovski, K. Geisel, M. Zanini, M. A. Fernandez-Rodriguez, V. V. Naik, A. Frutiger, W. Richtering, T. Ellenbogen, N. H. Voelcker and L. Isa, Nano Lett., 2016, 16, 157-163.

31 Z. Huang, N. Geyer, P. Werner, J. de Boor and U. Gösele, Adv. Mater., 2010, 23, 285-308. 